\title{
High Tolerance and Delayed Elastic Response of Cultured Axons to Dynamic Stretch Injury
}

\author{
Douglas H. Smith, ${ }^{1}$ John A. Wolf, ${ }^{1}$ Theresa A. Lusardi, ${ }^{2}$ Virginia M.-Y. Lee, ${ }^{3}$ and David F. Meaney ${ }^{2}$ \\ Departments of ${ }^{1}$ Neurosurgery, ${ }^{2}$ Bioengineering, and ${ }^{3}$ Pathology and Laboratory Medicine, University of Pennsylvania, \\ Philade/phia, Pennsylvania 19104-6316
}

Although axonal injury is a common feature of brain trauma, little is known of the immediate morphological responses of individual axons to mechanical injury. Here, we developed an in vitro model system that selectively stretches axons bridging two populations of human neurons derived from the cell line $\mathrm{N}$-Tera2. We found that these axons demonstrated a remarkably high tolerance to dynamic stretch injury, with no primary axotomy at strains $<65 \%$. In addition, the axolemma remained impermeable to small molecules after injury unless axotomy had occurred. We also found that injured axons exhibited the behavior of "delayed elasticity" after injury, going from a straight orientation before injury to developing an undulating

Throughout the world, traumatic brain injury is a leading source of mortality and disability, particularly of children and young adults (Kraus et al., 1994; Sosin et al., 1995). Axonal injury is a common pathology resulting from brain trauma, in which the extent of axonal pathology is thought to play a major role in the outcome (Adams et al., 1982, 1989; Graham et al., 1988; Povlishock, 1992). It is proposed that the initial event in traumatic axonal injury is the deformation, or strain, of axons as the result of inertial loading commonly induced during motor vehicle crashes, falls, and assaults (Adams et al., 1984; Thibault et al., 1990; Gennarelli, 1993; Grady et al., 1993). This mechanical deformation is thought to damage the neurofilament structure in the cytoskeleton of axons, causing regional compaction and/or impaired transport. As a result, accumulation of transport material induces regional axonal swelling (Povlishock et al., 1992). The primary changes in neurofilament structure may be augmented by ionic shifts and secondary activation of proteases to degrade the axonal ultrastructure further. Ultimately, axons may disconnect at the distal border of swollen regions, forming classic terminal bulb formations followed by Wallerian degeneration. Remarkably, this process has been observed to occur over the course of hours to even months after injury (Povlishock and Becker, 1985; Povlishock, 1993; Sherriff et al., 1994; Pierce et al., 1998). Although these processes of evolving axonal pathology have been well characterized in humans and several experimental models, little is known of the immediate events during and after dynamic deformation of axons. This information may help elucidate the

\footnotetext{
Received Dec. 8, 1998; revised Feb. 26, 1999; accepted March 17, 1999.

This work was supported by National Institutes of Health Grants AG12527 and NS08803 and by a grant from the Whitaker Foundation. We thank Brian Helmke and Peter Davies for their generous assistance with the deconvolution microscopy studies and Jeanne Marks for her excellent preparation of this manuscript.

Correspondence should be addressed to Dr. Douglas H. Smith, University of Pennsylvania, 3320 Smith Walk, 105 Hayden Hall, Philadelphia, PA 19104-6316. Copyright (C) 1999 Society for Neuroscience 0270-6474/99/194263-07\$05.00/0
}

course as an immediate response to injury, yet gradually recovering their original orientation. Surprisingly, some portions of the axons were found to be up to $60 \%$ longer immediately after injury. Subsequent to returning to their original length, injured axons developed swellings of appearance remarkably similar to that found in brain-injured humans. These findings may offer insight into mechanical-loading conditions leading to traumatic axonal injury and into potential mechanisms of axon reassembly after brain trauma.

Key words: axon; trauma; elasticity; dynamic deformation; tolerance; axolemmal permeability; neurofilament biomechanical factors that initiate the development of axonal pathology.

Central to understanding the induction of axonal pathology is defining the relationship between the applied mechanical force and the structural or functional response of the axon. For example, the mechanical threshold for primary axotomy (the severing of axons at the time of injury) is useful for developing preventative strategies for brain trauma. Current estimates of the mechanical tolerance for the structural and functional limit of in vivo axons of the CNS have been determined from ex vivo preparations using the squid giant axon (Galbraith et al., 1993), the frog sciatic nerve (Gray and Ritchie, 1954), the rat tibial nerve (Rydevik et al., 1990), and the pedal nerve of the slug (Jenkins and Carlson, 1904). These estimates have been complemented with data from physical and computational models (Zhou et al., 1994; Meaney et al., 1995) to develop a proposed tolerance standard of axons to mechanical damage. However, none of these studies have measured directly the pathological or mechanical changes associated with the deformation of intact CNS mammalian axons. In the present study, we examine the dynamic deformation of axons in vitro and report the threshold for primary axotomy, as well as novel findings on the acute temporal evolution of axonal response to stretch injury.

\section{MATERIALS AND METHODS}

Cell culture. We chose the N-Tera2 cl/D1 (NT2) cell line as our neuronal substrate because of the well-characterized ability of this cell line to differentiate into robust human neurons (Pleasure et al., 1992; Pleasure and Lee, 1993). In addition, this cell line has been shown to respond to excitatory injury in a manner similar to that of primary neuronal cell cultures (Munir et al., 1995). The NT2 cells were maintained in culture with OptiMEM (Life Technologies, Gaithersburg, MD) media supplemented with 5\% fetal bovine serum (FBS; HyClone, Logan, UT) and 1\% penicillin-streptomycin (Pen-Strep; Life Technologies). To differentiate the NT2 cells into neurons (NT2N), we cultured NT2 cells for 5 weeks 
in DMEM supplemented with 10\% FBS (HyClone), antibiotics (1\% Pen-Strep; Life Technologies), and $10 \mu \mathrm{M}$ retinoic acid (Sigma, St. Louis, $\mathrm{MO})$. To isolate neurons in the culture, we trypsinized the cells, triturated them with a fire-polished Pasteur pipette, and replated them in DMEM supplemented with 5\% FBS and mitotic inhibitors $(10 \mu \mathrm{M}$ 5-flouro-2'-deoxyuridine, $10 \mu \mathrm{M}$ uridine, and $1 \mu \mathrm{M}$ cytosine $\beta$-arabinofuranoside; Sigma) for $9 \mathrm{~d}$. The cells remaining after this procedure have been determined to be $99 \%$ neuronal. These NT2N neurons were seeded on a treated (poly-D-lysine, fibronectin, and Matrigel) deformable substrate (Specialty Manufacturing, Saginaw, MI) in custom-designed culture wells. A $1.5 \times 16 \mathrm{~mm}$ clear silicon barrier was placed on the membrane in the center of the well before plating of the NT2N cells to create a $1.5 \mathrm{~mm}$ "gap" through the center of the membrane. Cells were allowed to attach for $24 \mathrm{hr}$ before the barrier was removed. The temporary barrier prevents neurons from seeding in the gap region, creating a cell-free area for growth of isolated axons. After barrier removal, axons begin traversing the gap, ultimately synapsing with neurons on the other side (Fig. 1). The diameter of the axons crossing the gap ranged from 0.5 to $1.5 \mu \mathrm{m}$, typical widths of in vivo human axons. These cultures were maintained in conditioned media $(50 \%$ media from the first replate and $50 \%$ DMEM with 5\% FBS) for 3 weeks before an experiment, because it has been demonstrated previously that NT2N cells express a mature neuronal phenotype similar to that of in vivo human neurons with regard to receptor function by 2-2.5 weeks after plating (Munir et al., 1995).

Stretch device. The stretch device consisted of an aluminum cover block, a stainless steel plate with a machined $1.5 \times 18 \mathrm{~mm}$ slit, and an air pulse-generating system (Fig. 1). The culture well was inserted into the cover block and then placed on the slit plate so that the area of the deformable substrate contained the cultured axons. The cover plate was attached to the microscope stage, creating a sealed chamber. The top plate had a quartz viewing window in the center, an air inlet for compressed air, and a dynamic pressure transducer (Entran model EPXV01-25P-/16F-RF, Fairfield, NJ) to monitor internal chamber pressure. The introduction of compressed air into the chamber was gated by a solenoid (Parker General Valve, Elyria, $\mathrm{OH}$ ). The solenoid and the pressure transducer were controlled and monitored by an analog-todigital board (Keithley Metrabyte, Cleveland, OH) integrated with a computer data acquisition system (Capital Equipment Corporation, Billerica, MA). The device was mounted on the stage of a Nikon inverted microscope (Optical Apparatus, Ardmore, PA), allowing for continuous observation and photography of the axons throughout the experiments (Fig. 1).

Axonal stretch. A controlled air pulse was used to induce stretch to only the cultured axons traversing the gap in the well (Fig. 1). A rapid change in chamber pressure (rise time, $20 \mathrm{msec}$; duration, $50 \mathrm{msec}$ ) deflects downward only the portion of the substrate that contains the cultured axons; as a result, only these axons are stretched transiently to mimic the in vivo conditions of traumatic brain injury. Preliminary studies using microbeads attached to the substrate demonstrated that at static peak deflection, the axons remained attached to the membrane (data not shown). Therefore, membrane deflection correlated directly to uniaxial strain on the axons. Analysis of processes was constrained to axons within $\pm 10^{\circ}$ parallel to the major stretch axis. Measurement of nominal uniaxial strain $(\epsilon)$ was calculated by measuring the centerline membrane deflection $(\delta)$ and substituting into the geometric relationship:

$$
\varepsilon=\frac{w^{2}+4 \delta^{2}}{4 \delta w} \sin ^{-1}\left(\frac{4 \delta w}{w^{2}+4 \delta^{2}}\right)-1.0,
$$

if the measured deflection was less than one-half of the slit width $(w)$. If the measured deflection was greater than one-half of the slit width, then the applied stretch was calculated from a second geometric relationship:

$$
\varepsilon=\frac{w^{2}+4 \delta^{2}}{4 \delta w}\left(\pi-\sin ^{-1}\left(\frac{4 \delta w}{w^{2}+4 \delta^{2}}\right)\right)-1.0 .
$$

A strain value $(\epsilon)$ of 0.5 indicates that the axon was stretched to $50 \%$ above its initial length. For the experiments presented here, internal chamber pressure was 5-7 psi, correlating to a uniaxial strain on the axons of $0.58-0.77$, or $58-77 \%$ beyond its initial length. In all cases, the rate at which this strain was applied to the axon was between 26 and 35 $\mathrm{sec}^{-1}$, well within the range for traumatic injury. Over 50 separate experiments using these injury parameters were performed for this study.

Microscopy. Phase and fluorescent microscopy and photomicrography were performed on a Nikon Diaphot microscope with a Nikon 8008 camera. Confocal microscopy was performed with a Zeiss LSM5 (Hei- delberg, Germany). Deconvolution microscopy (Hiraoka et al., 1987) was performed on a Zeiss Axiovert 100 microscope equipped with a cooled CCD (Princeton Instruments, Trenton, NJ) and DeltaVision constrained iterative deconvolution software (Applied Precision, Issaquah, WA).

Measurement of morphological response to injury. In each experiment, we used time-lapse phase-contrast micrographs to measure the change in axonal geometry after stretch. Micrographs were recorded before and after stretch at regular intervals ( $2 \mathrm{~min}$ ) in a selected set of experiments. The micrograph set was transferred to digital format, and an image analysis program (ImagePro, West Chester, PA) was used to measure the length of the axonal processes within the field of view. Because a preliminary study showed that the axons did not detach from the membrane below the primary axotomy threshold, the axons appearing in the field before the stretch were also present at all times after stretch. Moreover, regular morphological markers on the axons in the field allowed the direct tracking of a portion of an axonal segment within the field of view. Therefore, these data reflected the true length change of the axonal process after a controlled stretch.

To quantify the morphological response over time, we defined a distortion (distension) parameter $\left[D\left(t_{i}\right)\right]$ that reflected the change in geometry from the initial axon segment length before stretch $\left(L_{\mathrm{o}}\right)$ to the length of the same segment at a time after stretch $\left[L\left(t_{i}\right)\right]$ :

$$
D\left(t_{i}\right)=L\left(t_{i}\right) / L_{\mathrm{o}} .
$$

By definition, before stretch the distortion of the process is 1.0 (i.e., there is no change in length). After the stretch, values of $D\left(t_{i}\right)$ increased above 1.0 because there was a net increase in axon segment length in these samples.

Immunohistochemistry. To evaluate the disruption of both the axonal cytoskeleton and the axonal transport after trauma, we used immunohistochemical techniques to stain neurofilament protein. The cultures were fixed $2 \mathrm{hr}$ after injury in $2 \%$ paraformaldehyde and $0.05 \%$ gluteraldehyde for $30 \mathrm{~min}$, permeabilized with $0.5 \%$ Triton $\mathrm{X}-100$ for $1 \mathrm{~min}$, and then labeled with monoclonal antibodies (RMO-281; 1:5) against phosphorylated neurofilament medium side arms (Lee et al., 1987). These antibodies were colocalized with fluorescent label conjugated to anti-mouse IgG (Alexa 488; Molecular Probes, Eugene, OR).

Axolemmal permeability. The permeability of axonal membranes (axolemma) after stretch injury was evaluated using Alexa 488 hydrazide (Molecular Probes), a membrane-impermeant fluorescent dye with a molecular weight of $570 \mathrm{Da}$. Accordingly, intracellular accumulation of this dye demonstrates the membrane permeability of small molecules. Axons were deformed with a strain of 60 or $75 \%$ in the presence of 200 $\mu \mathrm{l}$ of $580 \mu \mathrm{M}$ Alexa 488 hydrazide dissolved in control saline solution (CSS; $120 \mathrm{~mm} \mathrm{NaCl}, 5.4 \mathrm{~mm} \mathrm{KCl}, 0.8 \mathrm{~mm} \mathrm{MgCl}_{2}, 1.8 \mathrm{~mm} \mathrm{CaCl}_{2}, 15 \mathrm{~mm}$ glucose, and $25 \mathrm{~mm}$ HEPES, $\mathrm{pH} 7.4$, at $335 \mathrm{mOsm})$. The dye solution was immediately rinsed off after injury and replaced with CSS. The axons were then evaluated with phase and fluorescence microscopy and photographed at $5 \mathrm{~min}$ after injury as described above. As a positive control, the NT2N cultures were permeabilized with a single rinse of $0.005 \%$ Saponin (Sigma) in CSS, which was washed off with CSS, and then incubated for 2 min with dye solution. As a negative control, the cultures were incubated in the dye solution without injury or Saponin for $2 \mathrm{~min}$.

\section{RESULTS}

Using NT2N human neuron cultures (Pleasure et al., 1992; Pleasure and Lee, 1993), we developed an in vitro model system of isolated axonal stretch. This system uses an injury device that can precisely control the biomechanics of axon stretch (i.e., strain and strain rate) and can be integrated with an inverted microscope. Accordingly, we were able to observe continuously the acute temporal response of axons to uniaxial stretch at specific strains and strain rates.

\section{The threshold for primary axotomy in cultured neurons}

We found that the axons remained attached to the substrate until levels of strain inducing primary axotomy were reached. Examination of axons with phase microscopy during and immediately after stretch revealed that axons experienced strains that corresponded to the underlying substrate until primary axotomy occurred. We determined that the uniaxial strain on the substrate at 

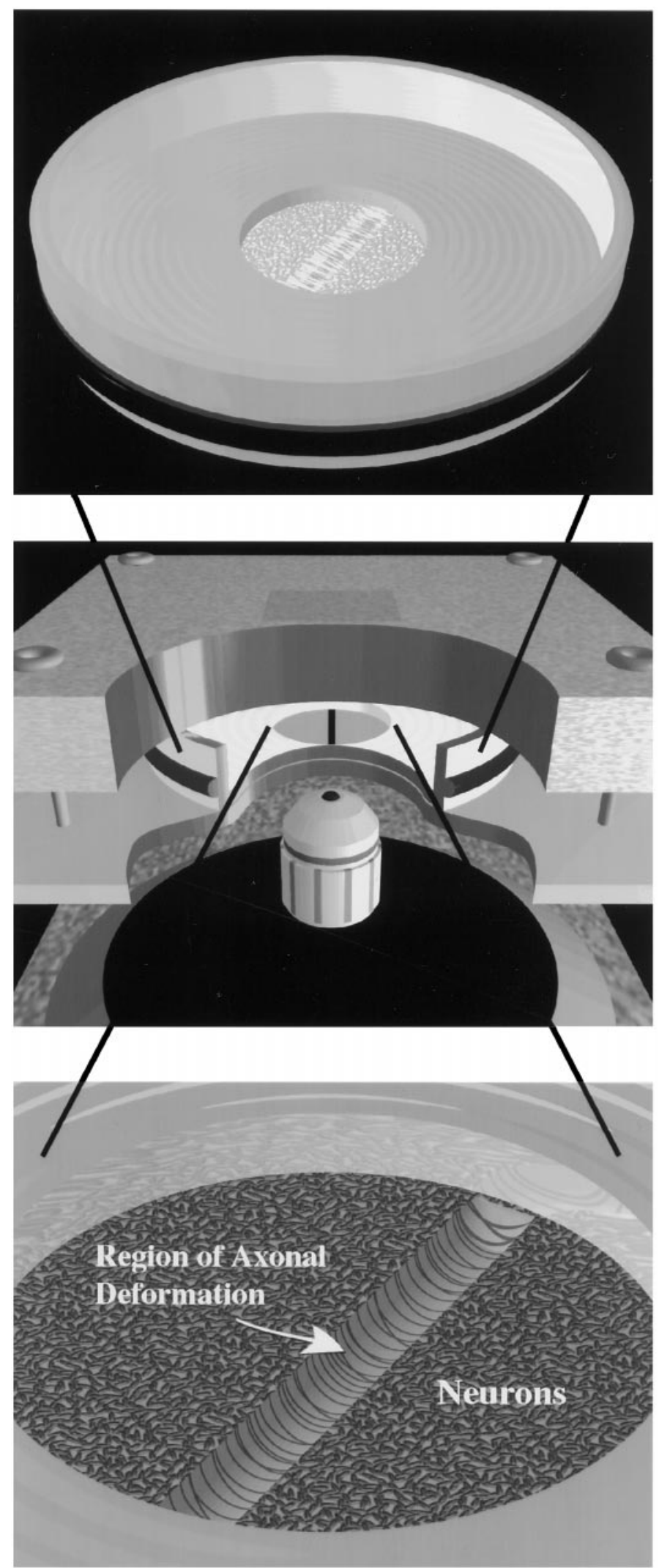

Figure 1. Schematic illustration of the technique and apparatus used to induce dynamic stretch injury to axons spanning two populations of human neurons. Top, Stainless steel well with a thin transparent deformable membrane in the center on which neurons (NT2N) are plated is shown. A $1.5 \times 18 \mathrm{~mm}$ cell-free gap is formed in the middle of the membrane that is bridged only by axons. Middle, The well is placed in the
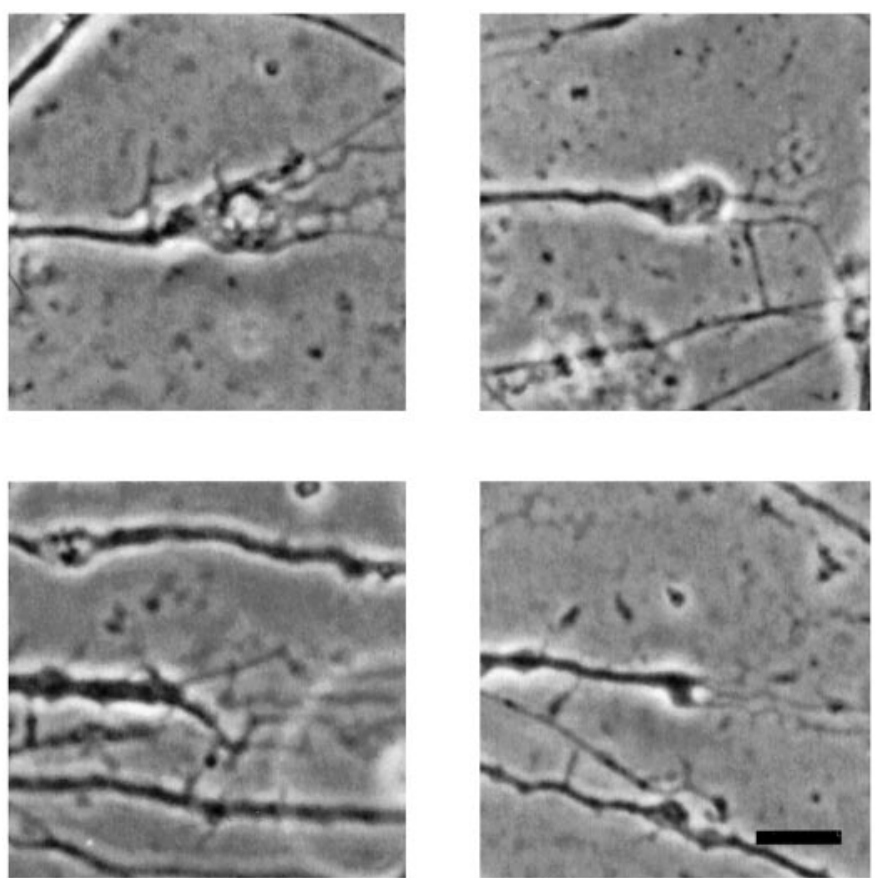

Figure 2. Phase-contrast photomicrographs of four examples of primary axotomy immediately after dynamic stretch injury. Primary axotomy, found only at strains $>65 \%$, is represented by severed axons shown in the middle of each panel. Note the torn or shredded appearance of the axonal terminal stumps with possible cytoskeletal remnants trailing out. Scale bar, $10 \mu \mathrm{m}$.

which primary axotomy could be found was $>65 \%$. However, even strains of $77 \%$ did not sever all axons. We also observed that primary axotomy resulted in the appearance of a shredded or torn terminal end of the severed axons (Fig. 2). Extruding from these ends were thin fibrils that may represent cytoskeletal remnants trailing out.

\section{The morphological response to stretch injury}

Below the level of strain for primary axotomy, axons maintained their overall position on the substrate but were consistently found to have undulating distortions at periodic points along their length immediately after trauma (Fig. 3). Although the extent and number of these distensions increased with an increase in the applied axonal strain, it is important to note that these distensions were consistently observed at all strains and strain rates used in the present study (i.e., $0.58-0.77$ strain; $26-35 \mathrm{sec}^{-1}$ strain rate). Independent of the shape or extent of distortion of the elongated regions, the axons gradually recovered their straightened prestretch shape over a period of $45 \mathrm{~min}$, with most of the relaxation occurring in the first $10 \mathrm{~min}$ (Fig. 3). It is important to note that the delayed elastic response of regions of injured axons was consistently observed in $>50$ separate experiments using the described techniques.

stretch device (cut away in the figure to reveal components) that consists of an aluminum cover block with a quartz viewing window and a stainless steel plate on the bottom with a machined $1.5 \times 18 \mathrm{~mm}$ slit that aligns with the gap region on the membrane. For continuous observation of the axons via an inverted microscope, the apparatus is bolted to the microscope stage that also creates a sealed chamber. Bottom, Compressed air is introduced into the chamber causing a downward deflection of only the gap region of the membrane, thereby inducing uniaxial stretch of the axons. 

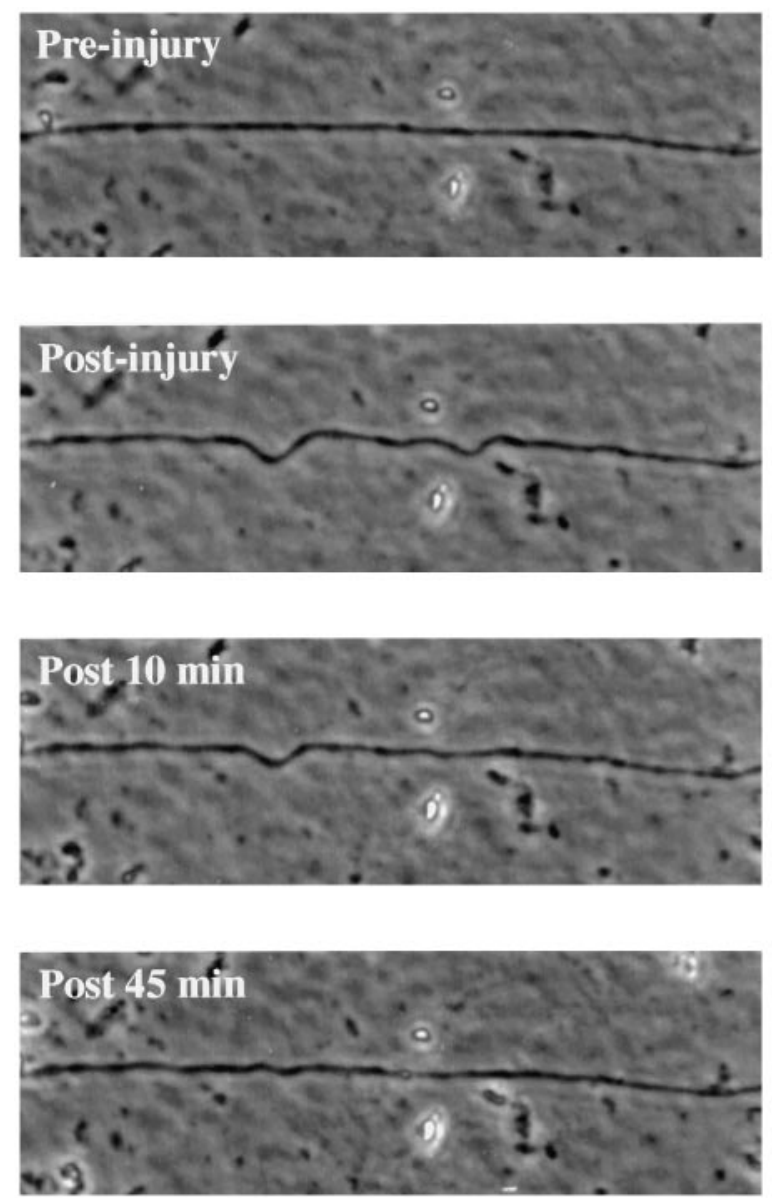
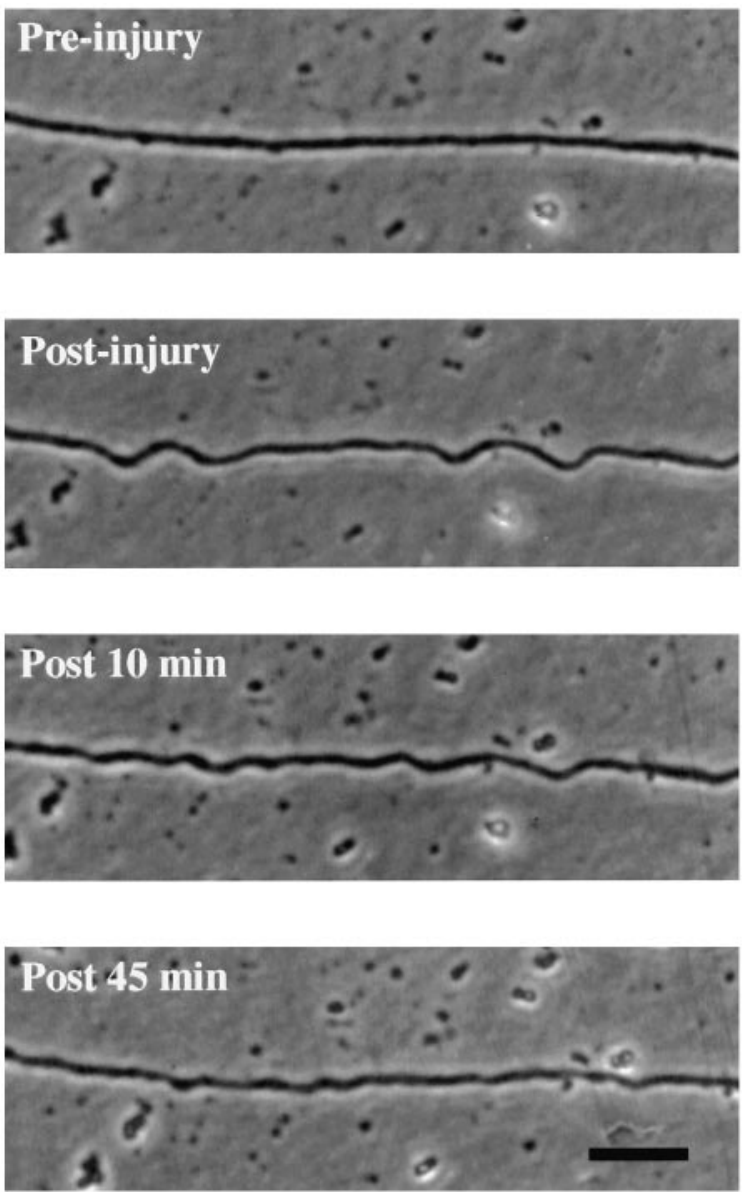

Figure 3. Phase-contrast photomicrographs of two examples (left, right) of the temporal evolution of the delayed elastic response of axons to dynamic stretch injury. As labeled, axons change from a straight orientation before injury to developing large undulations immediately at the time of injury yet gradually reassume their original orientation within $1 \mathrm{hr}$. Scale bar, $10 \mu \mathrm{m}$.

Because the periodic distortions occurred at selected points along the axon, we examined the regional geometric change in injured axons over time using serial photomicrographs to quantify the immediate increase and gradual recovery in length after stretch. Focusing on subregions of processes that showed geometric changes after stretch, we measured the regional distortion that occurred in these regions as a consequence of stretch over axon lengths of $\sim 200 \mu \mathrm{m}$. We found that the overall length increased almost $8 \%$ immediately after stretch and that the greatest reversal of this increase occurred within the first 5 min after injury. This increased average length remained significant until 30 min after injury (Fig. 4). However, although the total length increased $<8 \%$ after injury, the increase in length of many individual distortions (undulations) approached the level of the applied substrate stretch. For example, many of the local distortions had a half circle or triangle appearance immediately after injury (as illustrated in Fig. 3), in some cases measuring up to a remarkable $60 \%$ increase in length compared with their preinjury status. This observation is supportive evidence that the substrate strain was translated to the tissue. Taken together, these data also show the nonuniformity of the distortion phenomena of axons after dynamic stretch injury. Some axon regions appeared to have an elastic recovery after injury (i.e., immediately returning to their preinjury length), whereas other regions of the same axon demonstrated a delayed elastic response.

\section{Changes in neurofilament structure after stretch}

By $2 \mathrm{hr}$ after stretch injury, multiple swellings along the length of many axons could be observed with phase microscopy. We subsequently found that these swellings could also be consistently observed at $2 \mathrm{hr}$ after injury in fixed preparations of axons stained with antibodies targeting neurofilament sidearms. Confocal and deconvolution microscopy of these samples demonstrated that the accumulation of neurofilament in the axonal swellings appeared strikingly similar to swollen axons described in human brain injury. In addition, both confocal and deconvolution microscopy revealed that a central core of neurofilament was still apparent in some of these swellings represented by a more intense immunoreactivity to the neurofilament antibody. In some cases, this central core appeared disturbed from its original orientation with a tortuous course, even though the axon as a whole had returned to its preinjury orientation (Fig. 5).

\section{Axolemmal permeability after stretch injury}

With no stretch injury or chemical permeabilization, axons did not take up the 570 Da Alexa 488 dye (i.e., the axolemma was impermeant to small molecules) (Fig. 6A,B). However, chemical permeabilization of nonstretched axons did induce substantial uptake of the Alexa 488 dye (Fig. 6C,D). In comparison, 5 min after stretch injury to axons, a $60 \%$ strain (which produces no axotomy) did not induce uptake of dye into any axons, all of which 

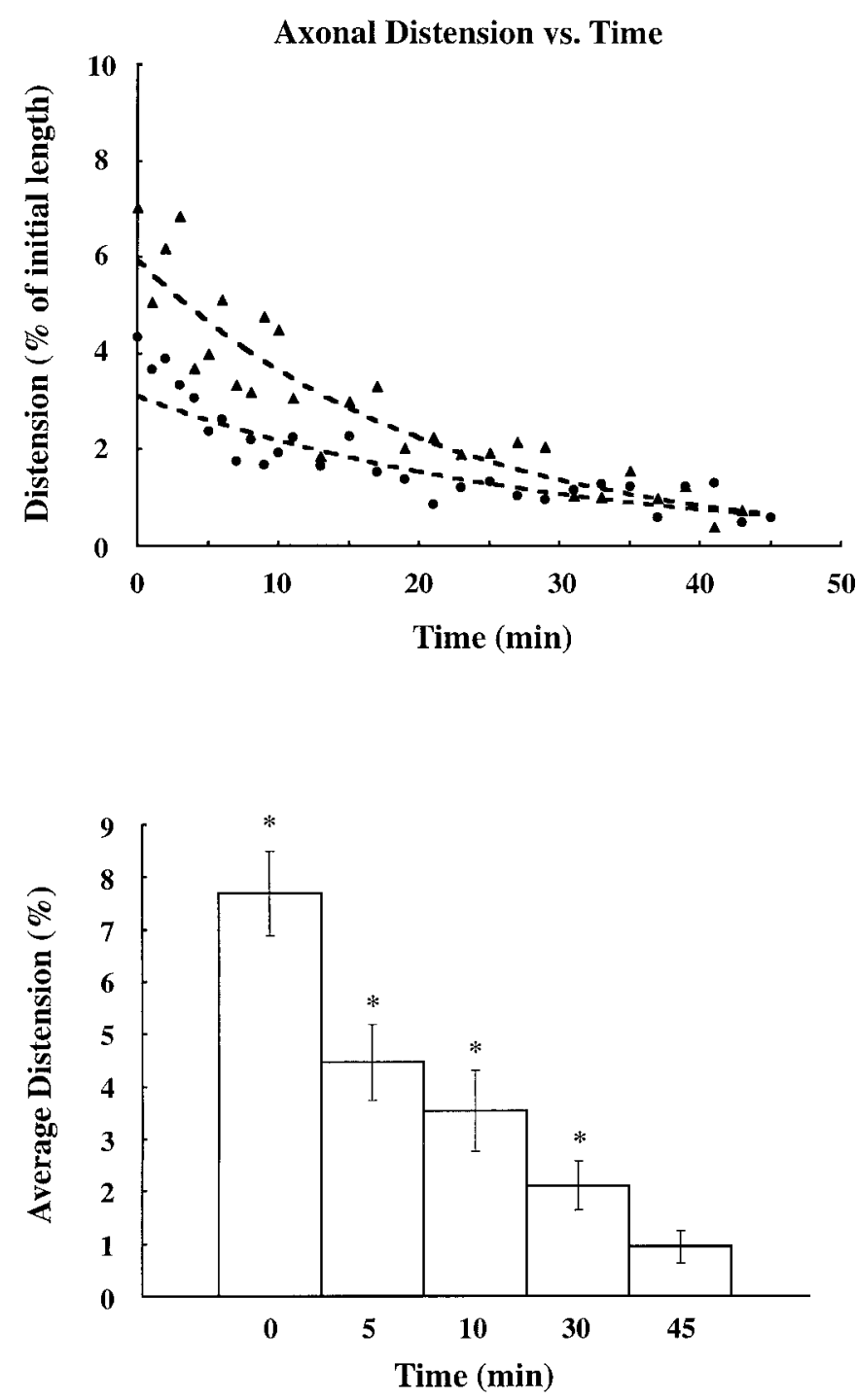

Figure 4. Quantitative temporal maps of the delayed elastic response of axons to dynamic stretch injury. Top, The temporal change in percent initial total length of two individual axons (circles, triangles) after stretch injury. Bottom, Average percent change in length of 11 axons over time after injury $\left({ }^{*} p<0.001\right.$ compared with preinjury length).

demonstrated the typical post-trauma undulated orientation (Fig. $6 E, F)$. At the strain of $75 \%$, there was modest uptake of dye, only in axons that had been severed (primary axotomy) (Fig. 6G,H). Axons not severed at these higher strains were not permeable to the dye. In severed axons, the extent of dye uptake appeared to be less than that for chemically permeabilized axons on the basis of the general intensity of the fluorescence signal. Therefore, after stretch injury, dye was not taken up by the axons unless axotomy had occurred.

\section{DISCUSSION}

In the present study we developed an in vitro model system that can deliver dynamic uniaxial deformation at specific strains and strain rates to axons spanning two populations of human neurons. The application of tensile strain to axons in this model, designed as a replication of the mechanical loading experienced by axons in vivo during traumatic brain injury, resulted in the temporal development of multiple foci of swelling along the axons. In these
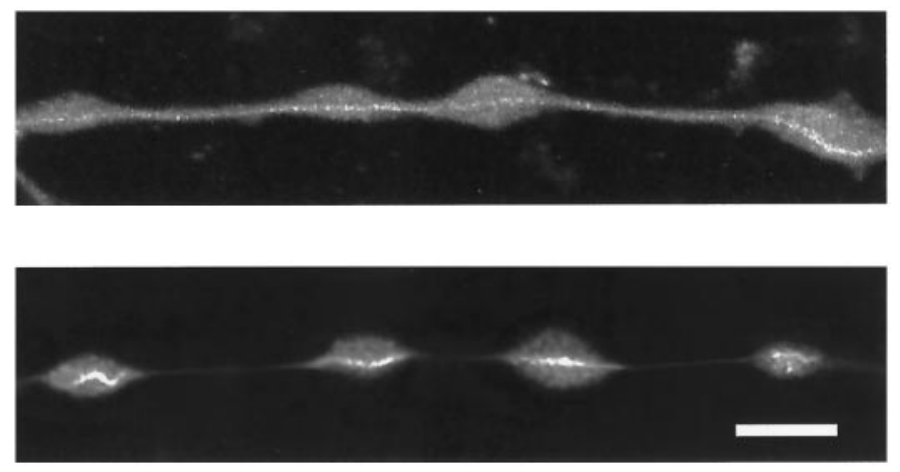

Figure 5. Accumulation of neurofilament in axons by $2 \mathrm{hr}$ after dynamic stretch injury. Confocal (top) and deconvolution (bottom) microscopy demonstrates multiple swellings along injured axons immunostained to reveal neurofilament protein. Both confocal and deconvolution microscopy reveals what appears to be a central core of neurofilament in the swellings represented by more intense immunostaining. In some of the swellings, this central core appears disturbed from its original orientation with a tortuous course, even though the axons as a whole had returned to their straight preinjury orientation.

axonal swellings, we found accumulation of neurofilament proteins, potentially demonstrating impaired axonal transport or local disassembly of the cytoskeleton. Notably, this pathology has a remarkably similar appearance of axonal swellings found after diff use brain injury in humans suffering brain trauma (Adams et al., 1989; Povlishock, 1992). Thus, this in vitro model seems to reproduce the most salient pathological feature of traumatic axonal damage found clinically. Because of our ability to control the applied stretch precisely and to observe directly the temporal evolution of pathology after stretch, three additional and potentially important observations were made. (1) Axons demonstrated a remarkably high tolerance to tensile strain even under dynamic loading conditions, with no primary axotomy observed from applied strains below $65 \%$, (2) axons exhibited the behavior of "delayed elasticity" after dynamic deformation, going from a straight orientation before injury to developing an undulating course immediately at the time of injury, yet gradually recovering their original orientation and morphology, and (3) despite the internal damage resulting from the stretch injury, the axolemma was not permeable to small molecules unless primary axotomy had occurred.

Previously, thresholds for primary axotomy were thought to be 25-30\% tensile strain when the axon was stretched in $<50 \mathrm{msec}$. Data from the tensile elongation of excised giant squid axons suggested that axons would structurally fail with strains of $\sim 25-$ $30 \%$ at strain rates in excess of $10 \mathrm{sec}^{-1}$ (Galbraith et al., 1993). Functional deficits, as measured by changes in resting membrane potential, appeared when dynamic strains were $>5 \%$. In peripheral nerve stretch studies using the dissected sciatic nerves from frogs, the structural failure limit was found at $33 \%$ at similar strain rates (Gray and Ritchie, 1954) and did not show changes in electrophysiological measures at levels 5-7\% strain in contrast to the squid giant axon. However, the axons used in these previous studies had relatively large diameters $(5-1000 \mu \mathrm{m})$, were not of mammalian origin, and were evaluated under the ex vivo conditions. Taken together, these potentially important considerations may have contributed to failure at much lower thresholds than that observed in the intact mammalian CNS axons used in the present study.

Evidence developed in parallel has suggested that the tolerance 

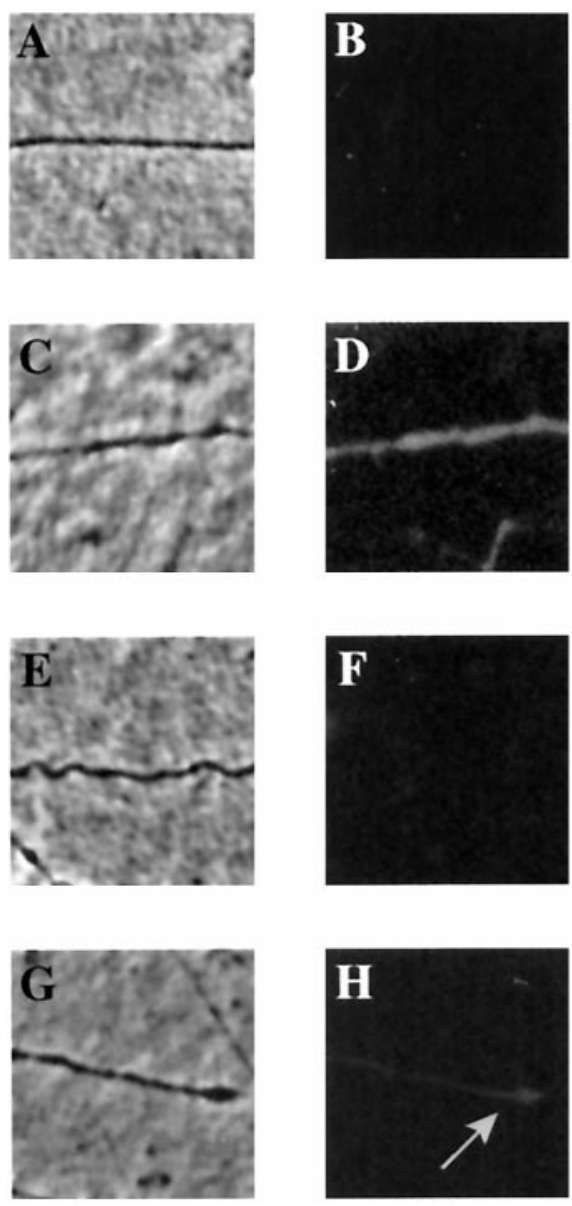

Figure 6. Representative images of axolemmal permeability shown under phase (left) and fluorescence (right) microscopy. These axons were exposed to a fluorescent dye, the uptake of which demonstrates the membrane permeability of small molecules. $A, B$, No dye is taken up in the absence of either stretch injury or chemical permeabilization of membranes. $C, D$, Dye is readily taken up after chemical permeabilization of the axolemma of axons. $E, F$, No dye is taken up by stretch-injured axons that are distorted but not severed. $G, H$, A detectable amount of dye is taken up in stretch-injured severed (primary axotomy) axons.

for primary axotomy is closer to the value reported herein rather than to previous data from squid and peripheral nerve studies. Recently, we developed a model of inertial brain trauma in pigs that produces selective injury to axons in the white matter (Smith et al., 1997). This model is accompanied by complementary physical and computational models used to determine the strain in regions shown to develop pathology (Zhou et al., 1994; Meaney et al., 1995). The estimated thresholds for shear strain associated with the appearance of axonal pathology (i.e., below the level of primary axotomy) in these collective models were between 15 and $24 \%$, higher than that reported to produce primary axotomy in earlier ex vivo studies. Our findings in the present study of primary axotomy tolerances of $65 \%$ tensile strain support our observations in the pig inertial brain trauma models of a substantially higher tolerance of axons than believed previously.

Our present results represent the first observation of a characteristic delayed elastic response of axons to dynamic deformation. The phenomena of delayed elasticity is commonly demonstrated in viscoelastic solids and is generally described as the complete recovery of shape for a material that has been deformed by a mechanical force (Flügge, 1975). The delayed elastic response of the axons to mechanical stretch in the present study included an immediate change in length of the axon and subsequent gradual recovery of the axon to its original length. Although it has been found previously that neurites demonstrate elastic responses under mechanical stress (Zheng et al., 1991), the ability of axons in the present study to resume original orientation gradually after potentially devastating deformation was unanticipated. Distortions of axons after brain trauma have been described at single time points after axonal trauma in animal models and in humans. However, it has been presumed that these distortions represented irreversible progression of cytoskeletal disruption and degeneration (Povlishock and Becker, 1985), underscoring the limitation of the in vivo studies to examine the temporal evolution of pathological changes in individual axons. We propose that our in vitro model replicates axonal distortions found after injury in vivo and demonstrates that, despite marked deformation, the axons can eventually regain their original (prestretch) geometry. The identification of a post-traumatic geometric change in the axon is an unprecedented phenomenon for neural tissue and may reflect a molecular-based process that can have important clinical implications in developing therapeutic strategies for traumatic axonal injury.

Although the source for the delayed elastic response of damaged axons is not presently clear, delayed elasticity is a well studied phenomenon in inert viscoelastic materials and is often attributed to one or more of the following factors: (1) the viscous flow of molecules in a network or dilute solution, (2) the selfdiffusion or "reptation" of molecules within a constraining molecular network, or (3) the active reformation of a molecular structure via new network connectivity (De Gennes, 1979). Because the axonal cytoskeleton has a high degree of interaction and cross-linking between its structural protein constituents (Burgoyne, 1991) and is a dynamically adaptable structure, these factors all may provide a basis for the mechanism(s) of delayed elasticity observed in the present study. Owing to the observation that axons return eventually to their original length after stretch, it seems that some part of the cytoskeleton is preserved sufficiently to provide an entropically driven restoring force. More precisely, the rate of recovery after stretch may be driven by the remaining elastic integrity of the network. Ongoing studies are evaluating the mechanical response of individual cytoskeletal constituents to mechanical deformation (Leterrier et al., 1996).

A major paradox of axonal injury found in vivo is the observation of extremely damaged axons among normal-appearing axons in the same white matter tracts after trauma. It is unclear why some axons regain their structure and function despite evidence of severe trauma, especially axons of similar size and orientation as that of neighboring axons that go on to degenerate. Although axonal bulb formation after axotomy is most likely an end stage event, it has not been resolved whether swollen yet still connected axons may recover. On the basis of our current results, we propose that axons have intrinsic mechanisms that facilitate recovery. The behavior of delayed elasticity in traumatized axons may reflect a "molecular memory" for axon shape such as has been posited for polymer dynamics (Sperling, 1992). Accordingly, cytoskeletal repair may be facilitated by further driving intrinsic molecular repair strategies to restore the initial cytoskeleton network geometry. Specifically, the viscoelastic reorientation of injured axons potentially brings back together regions separated by trauma and, thus, may improve the likelihood of cytoskeleton reassembly. Moreover, realignment of injured axons may help reinstate transport, which would further facilitate repair. It may 
also be important to consider that rather than being a detriment, the limited accumulation of neurofilament and other transport materials in damaged axons may actually provide the substrate for cytoskeletal reassembly after trauma.

Another potentially important finding in the axonal stretch model was that despite a $60 \%$ strain and substantial distortion of the axon, the axolemma appeared to remain impermeant to small molecules because no low molecular weight fluorescent dye was taken up in the cytosol. Modest uptake of dye into axons was only found at higher strains and only in axons that had been severed. These data suggest that substantial axolemmal permeability changes are not produced unless there is disconnection of the axon. These observations are consistent with the results of multiple previous studies using hematopoietic cells that have established the remarkable ability of the cell membrane to "flow" as an accommodation to substantial distention or deformations without changing permeability (Evans, 1973). In apparent contrast with these observations, studies of axonal damage in experimental animal models of brain trauma have demonstrated altered axolemmal permeability acutely after injury (Pettus et al., 1994; Povlishock et al., 1997). However, only a subpopulation of damaged axons was shown to have permeability changes in these animal studies. Therefore, changes in axolemmal permeability after trauma in animal models may also depend on the relative extent of axonal damage. Because contiguous regions of membranes rapidly adapt to high strain and distortions, the substantial and acute increases in axolemmal permeability shown in animal models of brain trauma models may reflect partial or complete axotomy, as demonstrated in the present study.

In summary, the present results suggest that axons not only have remarkably high resiliency to primary axotomy and axolemmal disruption from dynamic deformation but also demonstrate a unique ability to resume relatively normal shape and orientation. These findings may offer important considerations for the understanding of mechanical-loading conditions that lead to axonal injury. Moreover, these data may allow us to understand mechanisms of axon reassembly and regeneration after trauma using the model system described here.

\section{REFERENCES}

Adams JH, Graham DI, Murray LS, Scott G (1982) Diffuse axonal injury due to nonmissile head injury in humans: an analysis of 45 cases. Ann Neurol 12:557-563.

Adams JH, Doyle D, Graham DI, et al (1984) Diffuse axonal injury in head injuries caused by a fall. Lancet 2:1420-1422.

Adams JH, Doyle D, Ford I, Gennarelli TA, Graham DI, McClellan DR (1989) Diffuse axonal injury in head injury: definition, diagnosis, and grading. Histopathology 15:49-59.

Burgoyne P (1991) The neuronal cytoskeleton. New York: Wiley.

De Gennes P-G (1979) Scaling concepts in polymer physics. Ithaca, NY: Cornell UP.

Evans EA (1973) New membrane concept applied to the analysis of fluid shear- and micropipette-deformed red blood cells. Biophys J 13:941-954.

Flügge W (1975) Viscoelasticity. Berlin: Springer.

Galbraith JA, Thibault LE, Matteson RA (1993) Mechanical and electrical responses of the squid giant axon to simple elongation. J Biomech Eng 13-22.

Gennarelli TA (1993) Mechanisms of brain injury. J Emerg Med 11[Suppl 1]:5-11.

Grady MS, McLaughlin MR, Christman CW, Valadka AB, Fligner CL, Povlishock JT (1993) The use of antibodies targeted against the neurofilament subunits for the detection of diffuse axonal injury in humans. J Neuropathol Exp Neurol 52:143-152.

Graham DI, Adams JH, Gennarelli TA (1988) Mechanisms of nonpenetrating head injury. Prog Clin Biol Res 234:159-168.

Gray J, Ritchie J (1954) Effects stretch on single myelinated nerve fibers. Physiology 124:84-99.
Hiraoka Y, Sedat JW, Agard DA (1987) The use of a charge-coupled device for quantitative optical microscopy of biological structures. Science 238:36-41.

Jenkins O, Carlson A (1904) Physiological effects of the fluidity of the conducting substance in the pedal nerves of the slug-Ariolimax columianus. J Comp Neurol 14:85-92.

Kraus JF, McArthur DL, Silberman TA (1994) Epidemiology of mild brain injury. Semin Neurol 14:1-7.

Lee VMY, Carden MJ, Schlaepfer WW, Trojanowski JQ (1987) Monoclonal antibodies distinguish several differentially phosphorylated states of the two largest rat neurofilament subunits (NF-H and NF-M) and demonstrate their existence in the normal nervous system of adult rats. J Neurosci 7:3474-3488.

Leterrier JF, Kas J, Hartwig J, Vegners R, Janmey PA (1996) Mechanical effects of neurofilament cross-bridges. Modulation by phosphorylation, lipids, and interactions with F-actin. J Biol Chem 271:15687-15694.

Meaney DF, Smith DH, Shreiber DI, Bain AC, Miller RT, Ross DT, Gennarelli TA (1995) Biomechanical analysis of experimental diffuse axonal injury. J Neurotrauma 12:689-694.

Munir M, Lu L, McGonigle P (1995) Excitotoxic cell death and delayed rescue in human neurons derived from NT2 cells. J Neurosci 15:7847-7860.

Pettus EH, Christman CW, Giebel ML, Povlishock JT (1994) Traumatically induced altered membrane permeability: its relationship to traumatically induced reactive change. J Neurotrauma 11:507-522.

Pierce JES, Smith DH, Trojanowski JQ, McIntosh TK (1998) Enduring cognitive, neurobehavioral, and histopathological changes persist for up to one year following severe experimental brain injury in rats. Neuroscience 87:359-369.

Pleasure SJ, Lee VMY (1993) NTera 2 cells: a human cell line which displays characteristics expected of a human committed neuronal progenitor cell. J Neurosci Res 35:585-602.

Pleasure SJ, Page C, Lee VMY (1992) Pure, postmitotic, polarized human neurons derived from NTera 2 cells provide a system for expressing exogenous proteins in terminally differentiated neurons. J Neurosci 12:1802-1815.

Povlishock JT (1992) Traumatically induced axonal injury: pathogenesis and pathobiological implications. Brain Pathol 2:1-12.

Povlishock JT (1993) Pathobiology of traumatically induced axonal injury in animals and man. Ann Emerg Med 22:980-986.

Povlishock JT, Becker DP (1985) Fate of reactive axonal swellings induced by head injury. Lab Invest 52:540-552.

Povlishock JT, Erb DE, Astruc J (1992) Axonal response to traumatic brain injury: reactive axonal change, deafferentation, and neuroplasticity. J Neurotrauma 9:S189-S200.

Povlishock JY, Marmarou A, McIntosh TK, Trojanowski JQ (1997) Impact acceleration injury in the rat: evidence for focal axolemmal change and related neurofilament sidearm alteration. J Neuropathol Exp Neurol 56:347-359.

Rydevik BL, Kwan MK, Myers RR, Brown RA, Triggs KJ, Woo SL, Garfin SR (1990) An in vitro mechanical and histological study of acute stretching on rabbit tibial nerve. J Ortho Res 8:694-701.

Sherriff FE, Bridges LR, Gentleman SM, Sivaloganathan S, Wilson S (1994) Markers of axonal injury in postmortem human brain. J Neuropathol 88:433-439.

Smith DH, Chen X-H, Xu B-N, McIntosh TK, Gennarelli TA, Meaney DF (1997) Characterization of diff use axonal pathology and selective hippocampal damage following inertial brain trauma in the pig. J Neuropathol Exp Neurol 56:822-834.

Sosin DM, Sniezek JE, Waxweiler RJ (1995) Trends in death associated with traumatic brain injury, 1979 through 1992. JAMA 273:1778-1780.

Sperling LH (1992) Introduction to physical polymer science. New York: Wiley.

Thibault LE, Gennarelli TA, Margulies SS, Marcus J, Eppinger R (1990) The strain dependent pathophysiological consequences of inertial loading on central nervous system tissue. In: Proceedings of the International Conference on the Biomechanics of Impact, pp 191-202. Lyon, France.

Zheng J, Lamoureau P, Santiago V, Denner II, Buxbaum RE, Heidemann SR (1991) Tensile regulation of axonal elongation and initiation. J Neurosci 11:1117-1125.

Zhou C, Khalil T, King A (1994) Shear stress distribution in the porcine brain due to rotation impact. In: Proceedings of the 38th Stapp Car Crash Conf, pp 133-143. 